\title{
DESIGN OF ATOMIZERS AND BURNERS FOR COAL-WATER SLURRY COMBUSTION
}

Grant Number: DE-FG22-95PC95105

Progress Report for Period 1/1/97- 3/31/97

\author{
A. Mansour, and N. Chigier \\ Spray Systems Technology Center \\ Dept. of Mechanical Engineering, Carnegie Mellon University \\ Pittsburgh, PA 15213
}

\begin{abstract}
A review of the results of the current period is included. Section 1 summarizes the progress that was made during the current period. Section 2 discusses the activities planned for the next period. Section 3 summarizes the theory that was developed on the capillary flow within long filaments of liquid during the breakup of ligaments of Newtonian and Non-Newtonian Fluids.
\end{abstract}

\section{CURRENT PERIOD PROGRESS REPORT}

The current period was mainly dedicated to developing a theory to account for the flow into the droplet and back into the droplet attachment region in the falling droplet experiments. Six samples of coal water slurries were also tested for determination of the extensional viscosity using the falling droplet technique. A spray chamber was built to study the breakup of coal water slurry samples provided by Penn State.

Measurement of the extensional viscosity of coal water slurries

Penn State provided 6 samples of coal-water slurry. All samples were subjected to screening to determine if the extensional viscosity may play an important role in atomization. An attempt has been made to measure the extensional viscosity of the coal-water slurry samples provided by Penn State using the falling droplet technique. The samples solid loading ranged from 50.0 to 54.6 of coal by mass. The shear viscosity at a shear rate of $100 \mathrm{sec}-1$ ranged from 109 to $714 \mathrm{cp}$. The attempt failed however because the coal water slurry samples did not generate a trailing filament.

All samples were observed in the drip mode of breakup. The samples were placed in a capillary tube with an inner diameter of $1.1 \mathrm{~mm}$. As the droplets fell from the end of the capillary tube, digital images were obtained with the Greenfield Digital Spray Analyzer. The images from the CCD camera of the Greenfield were stored in a computer and translated into a common personal computer image for presentation. The filament life time was less than $20 \mathrm{~ms}$ indicating that elastic behavior is not 
dominant. Typically only viscoelastic liquids and liquids which show predominant elastic behavior generates a trailing filament. This indicates that for the samples provided the extensional viscosity did not play any significant role. Possibly the stabilizer concentration was too low to affect the breakup of the coal-water slurry samples.

\section{ACTIVITIES PLANNED FOR NEXT PERIOD}

Penn State provided a Delavan atomizer for characterization under quiescent conditions (no secondary and tertiary air flow) using the laser Diffraction particle sizer. In the past few weeks we have built a spray chamber to be used for characterization of the Delavan atomizer. In the coming months we will use all the 6 coal water slurry samples provided by Penn State and study the near and far fields of the atomizer. We will vary CWS flow rate, the atomizing air flow rate and study the effects of these parameters on breakup on the coal water slurry samples. We will perform the following tasks:

- Measure the coal-water slurry spray characteristics: particle size distributions using the laser diffraction particle analyzer.

- Study the effects of additives (particularly stabilizers), typically used in coal -water slurry fuels for their influence on rheological properties and atomization. Determine the influence of shear viscosity on atomization.

- Study the fundamental mechanisms of atomization of coal-water slurries. Develop models for the breakup of coal-water slurries using air-assist atomizers.

- Use the Malvern laser diffraction instrument for measurement of droplet size in the spray field produced by the triple concentric air assist atomizer.

- Vary flow rates of liquid and atomizing air. Measure drop size distributions. Determine quality of atomization from microphotography. Establish the relationships between rheological properties of solutions at high shear rates and atomization quality. 


\title{
3. PUBLICATIONS
}

The following is a copy of a manuscript showing the theory that we have developed on the breakup of capillary jets during the drip mode. This paper addresses the deficiencies of the falling droplet technique for measurement of extensional viscosity. Theoretical analysis of the fluid flow inside the filaments is presented to explain the discrepancies between length and diameter measurements (see our report of Grant Number: DE-FG22-95PC95105 Progress Report for Period $7 / 1 / 96-9 / 31 / 96)$.

\section{On the Capillary Flow within Long Filaments of Liquid During the Drip Mode of Breakup of Ligaments of Newtonian and Non- Newtonian Fluids.}

\author{
Y. Tambour, A. Mansour, and N. Chigier \\ Spray Systems Technology Center \\ Dept. of Mechanical Engineering, Carnegie Mellon University \\ Pittsburgh, PA 15213
}

\begin{abstract}
A theoretical analysis of the quasi-steady capillary flow within long filaments of liquid during the drip mode of breakup of Newtonian and Non-Newtonian fluids is presented. A polynomial solution for the capillary velocity profile of the liquid at the edge of the filament is employed. The coefficients in the polynomial solution are found here via satisfying the flow boundary conditions and the longitudinal momentum equation. In this way the solution for the velocity profile is expressed in terms of the longitudinal pressure differential. Future experiments in which the diameters at the center and the edge of the filament will be simultaneously measured during the stretching motion may serve for validation of the present solutions.
\end{abstract}

\section{Introduction}

Photographic visualization by Mansour, Chigier and Hopkins (1997) of the breakup of viscoelastic materials (aqueous solutions of Polyacrilamide E10) in the drip mode have shown that the ligaments 
of these polymer solutions undergo a very large stretching motion before they break up, resulting in long liquid filaments attached to the droplets. In order to extract information on the extensional properties of these fluids Mansour, Chigier and Hopkins (1997) measured the changes in filament diameter and length as a function of time by employing laser techniques. The rate of change of the filament diameter does not match the rate of change of the filament's length, suggesting that the volume of the filament does not remain constant during the stretching but rather decreases. This decrease in the filament's volume indicates that some of the liquid mass leaves the filament via a capillary flow through the edges of the filament. Additional experimental evidence to support this observation is the necking phenomenon at the center of the filament shown in the above mentioned photographic visualization. Due to surface tension forces, the small diameter at the center of the filament results in a larger pressure build-up at the center than at the edges of the filament where the diameter is larger. Thus, the pressure differential between the center and the edges of the filament serves as a driving force for the capillary flow within the filament that carries mass that leaves the filament throughout its edges.

In this paper, we present a theoretical analysis of the above observed capillary flow within long filaments of liquid during the drip mode of breakup of Newtonian and Non-Newtonian fluids. A polynomial solution for the capillary velocity profile of the liquid at the edge of the filament is employed. The coefficients in the polynomial solution are found here via satisfying the flow boundary conditions and the longitudinal momentum equation. In this way the solution for the velocity profile is expressed in terms of the longitudinal pressure differential. The solution for the flow rate of mass that is leaving the filament is obtained via integration of the velocity profile.

Future experiments in which the diameters at the center and the edge of the filament will be simultaneously measured during the stretching motion may serve for validation of the present solutions. The longitudinal pressure gradient can be extracted from the diameter differential between the center and the edge of the filament. By knowing the magnitude of the longitudinal pressure gradient the solution for the velocity profile is fully determined and so is the mass flow rate of liquid that leaves the filament. The theoretically predicted mass flow rate may then be compared with the mass flow rate that is extracted from the experimental data via the difference between the rate of radius shrinkage and the rate of length increase. Thus, the present theoretical study compliments the experimental observations and may serve as a useful tool in future simulations of the breakup mechanism and the formation of sprays of polymer solutions.

\section{Indirect Experimental Evidence}


In the present section, the indirect evidence that supports the assumption of mass flow that leaves a stretching liquid filament will be discussed as follows. An example of the drip mode breakup is shown in Figure 1. A long liquid filament is formed and stretched when a drop which is released from a capillary tube travels downward under the force of gravity.

The length of the filament is denoted by $\mathrm{L}$. The maximum radius and the radius of the neck are denoted by $R$ and $R_{N}$, respectively. All the above properties are functions of time t.

The volume of the filament is given by

$$
V(t)=\int_{0}^{L} R(z) 2 \pi r d r d z
$$

where the length $\mathrm{L}$, the radius $\mathrm{R}(\mathrm{z})$, and the volume $\mathrm{V}$ are all functions of time.

Since the function describing the change of the radius with the longitudinal distance $z, R(z)$, in unknown, one may assume either a conical shape for which the instantaneous volume of the filament is given by

$$
V(t)=\frac{1}{3} \pi\left[R^{2}(t)+R_{N}^{2}(t)+R(t) R_{N}(t)\right] L(t)
$$

or one may assume a cylindrical shape for the case that $\left(R-R_{N}\right) \rightarrow 0$, thus:

$$
V(t)=\pi R^{2}(t) L(t)
$$

The time derivative of Eq. (2b) yields:

$$
\frac{\dot{V}}{V}=2 \frac{\dot{R}}{R}+\frac{\dot{L}}{L}
$$

The first term on the RHS of Eq. (3) is negative. Thus, for a liquid filament of a constant volume, the two terms on the RHS of Eq. (3) cancel out and result in $\dot{V}=0$. However, experimental data by Mansour, Chigier and Hopkins (1997) indicate that (see Table 1):

$$
\left.\left|\frac{2 \dot{R}}{R}\right|\right\rangle \frac{\dot{L}}{L}
$$

and hence

$$
\dot{V} \angle 0
$$


that is, the volume of the filament decreases with time, indicating that some mass flows out of the filaments throughout its edges during the stretching motion. Thus, in the next section a theoretical analysis of this mass flow rate is presented.

Table 1: Change in volume and time derivatives of the length and the average radius during stretching of liquid filaments of aqueous solutions of Polyacrilamide E10. (Extracted from experimental data by Mansour, Chigier and Hopkins, 1997.)

Mass Conc. of

Polyacrilamide E10: $\quad 2 \dot{R} I R\left[s^{-1}\right] \quad \dot{L} I L\left[s^{-1}\right] \quad \dot{V} I V$

$\begin{array}{llll}1.0 & -1.73 & 0.89 & -0.84\end{array}$

$\begin{array}{llll}0.5 & -2.72 & 1.37 & -1.35\end{array}$

$\begin{array}{llll}0.25 & -5.35 & 2.76 & -2.59\end{array}$

$\begin{array}{llll}0.125 & -7.36 & 4.05 & -3.31\end{array}$

$\begin{array}{llll}0.0625 & -7.62 & 5.24 & -2.38\end{array}$

$\begin{array}{llll}0.03125 & -10.99 & 6.80 & -4.19\end{array}$

0.015625

$-23.18 .79-14.31$

0.0078125

$-42.813 .31 \quad-29.49$

0.003906

$-98.319 .92 \quad-78.38$

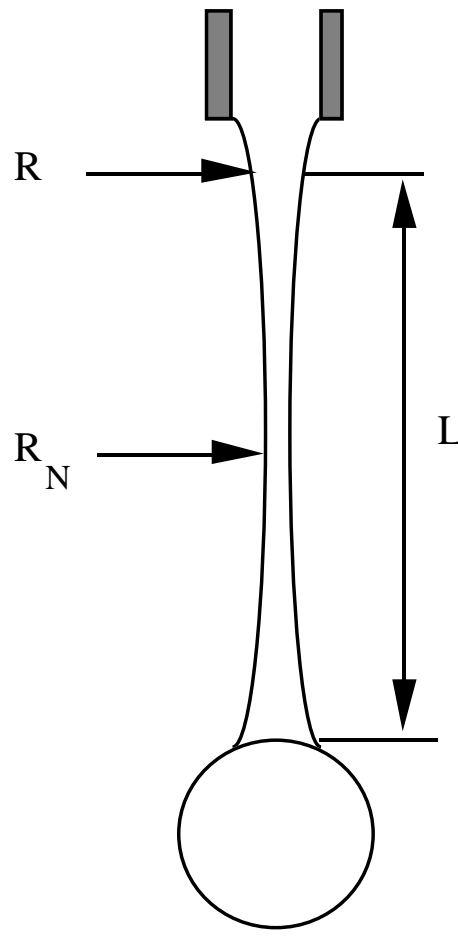

Fig. 1: Schematics of a stretching liquid filament. All dimensions are functions of time. 


\section{Theoretical Analysis}

For a quasi-steady, fully developed capillary flow at the edges of the filament, the momentum equation in the longitudinal $\mathrm{z}$ direction may be written as

$$
\frac{\partial p}{\partial z}=\frac{1}{r} \frac{\partial}{\partial r}\left(r \tau_{r z}\right)
$$

where $\mathrm{p}$ is the pressure, $\mathrm{r}$ is the radial coordinate in a cylindrical coordinate system, and $\tau_{r z}$ is the shear stress.

When analyzing Newtonian fluids, the shear stress is given by

$$
\tau_{r z}=-\mu \frac{\partial u}{\partial r}
$$

where $\mu$ is the Newtonian shear viscosity and $\mathrm{u}$ is the velocity in the longitudinal direction $\mathrm{z}$.

On the other hand, for Non-Newtonian fluids the Ostwald-deWaele Model is used here. This power law model is described by the following relation between the velocity gradient and the shear stress:

$$
\tau_{r z}=-\bar{\mu}\left|\frac{\partial u}{\partial r}\right|^{n-1} \frac{\partial u}{\partial r}
$$

where the values for the power " $n$ " may be found in Metzner (1956). It should be noted that for $n=1$ the Non-Newtonian model reduces to the Newtonian one with the viscosity $\bar{\mu}=\mu$.

The solution of Eq. (6) is not as straight forward as may seem at first sight since the diameter of the liquid filament decreases with time and this radial shrinkage is accompanied by a radial inwards flow. Thus, the longitudinal pressure gradient $\frac{\partial p}{\partial z}$ is an unknown function of $r$.

In order to overcome the above described difficulties, a polynomial solution is suggested here for the longitudinal velocity $u$ at the edges of the liquid filament. Denoting the radius at the edge of the liquid filament by $\mathrm{R}$ we express $u$ in the following polynomial form:

$$
u=u_{\max }\left[1-\sum_{i=1}^{m} A_{i}\left(\frac{r}{R}\right)^{i}\right]
$$

where $\mathrm{i}$ are positive integers and where $u_{\max }$ and the coefficients of $A_{i}$ are to be determined via satisfying the boundary conditions for the velocity $u$ and by satisfying the momentum equation (Eq. 6.) 
Obviously the number of terms, $\mathrm{m}$, is limited to the number of boundary conditions to be satisfied, and although in the present analysis we will use only three terms (i.e. $m=3$ ) for the sake of generality of the present theory we will carry on with the general notation $\mathrm{m}$.

The boundary conditions to be satisfied are as follows:

$$
\text { at } \mathrm{r}=0: \quad \frac{\partial u}{\partial r}=0
$$

and at $\mathrm{r}=\mathrm{R}$ :

$\mathrm{u}=0$

$$
\text { and } \quad \frac{\partial u}{\partial r}=0
$$

In the above, Eq. (10) is due to symmetry. In writing Eq. (11b) we assume that the shear stresses between the liquid filament and the surrounding air are very small and therefore may be neglected. The boundary condition given by Eq. (11a) assumes that if there is a non-zero longitudinal velocity at the circumference of the liquid filament, then it is included in the stretching rate of the filament as a whole, i.e. in the rate of change of the length of the filament as a function of time. Thus, $u$ represents the velocity of liquid that leaves the filament and the velocity of the stretching motion of the filament itself is not included in $u$.

Next, we satisfy the boundary condition given by Eq. (10). To do so, we first carry out the derivative of Eq. (9) with respect to $\mathrm{r}$ :

$$
\frac{\partial u}{\partial r}=u_{\max } \frac{1}{R} \sum_{i=1}^{m} i A_{i}\left(\frac{r}{R}\right)^{i-1}
$$

and observe that the aforementioned boundary condition (Eq. 10) is satisfied for

$$
A_{1}=0
$$

Thus, we rewrite the velocity profile, Eq. (9), and its derivative, Eq. (12), after resetting the starting point for the summations to be $i=2$ (instead of $i=1$ ). That is:

$$
u=u_{\max }\left[1-\sum_{i=2}^{m} A i\left(\frac{1}{R}\right)^{i}\right]
$$

and

$$
-\frac{\partial u}{\partial r}=u_{\max } \frac{1}{R} \sum_{i=2}^{m} i A_{i}\left(\frac{r}{R}\right)^{i-1}
$$


This intermediate step is presented here since we would like to show in what follows that upon satisfying the longitudinal momentum equation for a Newtonian fluid the volume flow rate out of the filament is proportional to the fourth power of the radius $R^{4}$, irrespective of the powers $\mathrm{i}$ and the magnitude of $\mathrm{m}$ in the summation. In this respect, it should be emphasized here that the only condition imposed on Eq. (14) is the requirement that the velocity profile be symmetrical with respect to the longitudinal axis $\mathrm{z}$.

Thus, we continue and carry out the following derivative:

$$
-\frac{1}{r} \frac{\partial}{\partial r}\left(r \frac{\partial u}{\partial r}\right)=u_{\max } \frac{i}{R^{2}} \sum_{i=2}^{m} i^{2} A_{i}\left(\frac{r}{R}\right)^{i-2}
$$

which is then substituted in the longitudinal momentum equation (Eq. 6) for a Newtonian fluid (see Eq. 7) of a constant viscosity $\mu$ to yield:

$$
-\frac{\partial p}{\partial r}=\mu u_{\max } \frac{i}{R^{2}} \sum_{i=2}^{m} i^{2} A_{i}\left(\frac{r}{R}\right)^{i-2}
$$

Next, one should note that the term resulting from $i=2$ is not a function of $r$ (since the power of $r$ is zero) and thus $u_{\max }$ can be evaluated as a function of the longitudinal pressure gradient at $\mathrm{r}=0$

$$
u_{\max }=\frac{R^{2}}{4 A_{2} \mu}\left(-\frac{\partial p}{\partial z}\right)_{r=0}
$$

Finally, the velocity profile $u$ is obtained via substituting Eq. (18) into

Eq. (14):

$$
u=\frac{R^{2}}{4 A_{2} \mu}\left(-\frac{\partial p}{\partial z}\right)_{r=0}\left[1-\sum_{i=2}^{m} A_{i}\left(\frac{r}{R}\right) i\right]
$$

and the volume flow rate out of the filament is given by:

$$
\dot{Q}=2 \pi \int_{0}^{R} u r d r=\frac{\pi R^{4}}{2 A_{2} \mu}\left(\frac{\partial p}{\partial z}\right)_{r=0}\left[\frac{1}{2}-\sum_{i=2}^{m} \frac{A_{i}}{i+2}\right]
$$

Thus, for a Newtonian fluid all coefficients $A_{i}$ (i=2,3,..m) may be lumped into one unknown coefficient $\mathrm{C}$ 


$$
\frac{1}{C}=\frac{\pi}{2 A_{2}}\left[\frac{1}{2}-\sum_{i=2}^{m} \frac{A_{i}}{i+2}\right]
$$

allowing the volume flow rate $\dot{Q}$ to be reexpressed as

$$
\dot{Q}=\frac{R^{4}}{\mu C}\left(-\frac{\partial p}{\partial z}\right)_{r=0}
$$

for which the coefficient $\mathrm{C}$ could be determined from experimental data in which all other properties in

Eq. (22) are provided.

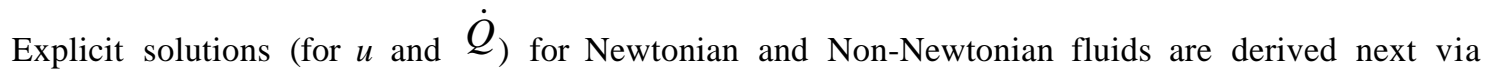
satisfying boundary conditions (11a) and (11b). After $A_{1}$ is determined via satisfying Eq. (10), the two additional boundary conditions (11a) and (11b) enable us to determine two additional unknowns $A_{2}$ and $A_{3}$.

Employing Eq. (14) and (15) for $\mathrm{m}=3$, one obtains:

$$
\begin{aligned}
& 1-A_{2}-A_{3}=0 \\
& 2 A_{2}+3 A_{3}=0
\end{aligned}
$$

which yields $A_{2}=3$ and $A_{3}=-2$

Thus for a Newtonian fluid the solution for the velocity profile becomes, (see Eq. 19)

$$
u=\frac{R^{2}}{12 \mu}\left(-\frac{\partial p}{\partial z}\right)_{r=0}\left[1-3\left(\frac{r}{R}\right)^{2}+2\left(\frac{r}{R}\right)^{3}\right]
$$

for which volume mass flow rate $\dot{Q}_{\text {becomes }}$

$$
\dot{Q}=\frac{\pi R^{4}}{80 \mu}\left(-\frac{\partial p}{\partial z}\right)_{r=0}
$$

In terms of $\left(-\frac{\partial p}{\partial z}\right)$ at $\mathrm{r}=\mathrm{R}$ one obtains:

$$
u=\frac{R^{2}}{6 \mu}\left(-\frac{\partial p}{\partial z}\right)_{r=R}\left[1-3\left(\frac{r}{R}\right) 2+2\left(\frac{r}{R}\right)^{3}\right]
$$


and $\quad \dot{Q}=\frac{\pi R^{4}}{40 \mu}\left(-\frac{\partial p}{\partial z}\right)_{r=R}$

For a Non-Newtonian fluid (see Eqs. 8 and 15) (29)

$$
\begin{array}{r}
\tau_{r z}=-\bar{\mu}\left[u_{\max } \frac{1}{R}\right]^{n}\left|6\left(\frac{r}{R}\right)^{2}-6\left(\frac{r}{R}\right)\right|^{n}\left[6\left(\frac{r}{R}\right)^{2}-6\left(\frac{r}{R}\right)\right] \\
u=\frac{R^{1+n}}{(6 \bar{\mu})^{\frac{1}{n}}}\left[-\frac{\partial p}{\partial z}\right]_{r=R}^{\frac{1}{n}}\left[1-3\left(\frac{r}{R}\right)^{2}+2\left(\frac{r}{R}\right)^{3}\right]
\end{array}
$$

for which

$$
\dot{Q}=0.15 \frac{\pi R^{3+n}}{(6 \bar{\mu})^{\frac{1}{n}}}\left(-\frac{\partial p}{\partial z}\right)^{\frac{1}{n}} r=R
$$

Note that for a Newtonian fluid i.e. for $\mathrm{n}=1$ Eqs. (30) and (31) reduce to Eqs. (27) and (28), respectively.

\section{REFERENCES}

1. Mansour, A., Chigier, N. and Hopkins, J., "Measurement of the Extensional Viscosity of Polyacrilamide Solutions by Uniaxial Stretching of Fluid Filaments," Proceedings of ILASSAmericas, Ottawa, Canada, 1997.

2. Metzner, A.B., "Advances in Chemical Engineering," Vol. I., Academic Press, New York, 1956, p. 103. 